RUNNING HEAD: Identity Content in Context

An Integrated Developmental Model for Studying Identity Content in Context

Renee V. Galliher

Utah State University

Renee.Galliher@usu.edu

Kate C. McLean

Western Washington University

Kate.McLean@wwu.edu

Moin Syed

University of Minnesota

moin@umn.edu

In press

Developmental Psychology

Version: 10/15/17 


\begin{abstract}
Historically, identity researchers have placed greater emphasis on processes of identity development (how people develop their identities) and less on the content of identity (what the identity is). The relative neglect of identity content may reflect the lack of a comprehensive framework to guide research. In this article, we provide such a comprehensive framework for the study of the content of identity, including four levels of analysis. At the broadest level, we situate individual identity within historical, cultural, and political contexts, elaborating on identity development within the context of shifting cultural norms, values, and attitudes. Histories of prejudice and discrimination are relevant in shaping intersections among historically marginalized identities. Second, we examine social roles as unique and central contexts for identity development, such that relationship labels become integrated into a larger identity constellation. Third, domains of individual or personal identity content intersect to yield a sense of self in which various aspects are subjectively experienced as an integrated whole. We explore the negotiation of culturally marginalized and dominant identity labels, as well as idiosyncratic aspects of identities based on unique characteristics or group memberships. Finally, we argue that the content of identity is enacted at the level of everyday interactions, the "micro-level" of identity. The concepts of identity conflict, coherence, and compartmentalization are presented as strategies used to navigate identity content across these four levels. This framework serves as an organizing tool for the current literature, as well as for designing future studies on the identity development.
\end{abstract}

Keywords: identity content; intersectionality 


\section{An Integrated Developmental Model for Studying Identity Content in Context}

Identity development is a psychosocial task with life-long relevance that has critical implications for health and well-being (for reviews see McAdams \& McLean, 2013; Meeus, 2011; Schwartz et al. 2013); indeed, knowing who one is may be one of the most fundamental components of being human. Given the importance of this task, there has been a tremendous amount of empirical attention paid to the topic across disciplines within psychology. Within the study of identity development, in particular, researchers have largely emphasized processes of identity development (how people develop their identities) and have placed relatively little emphasis on the content of identity (what the identity is; cf., Archer, 1985; Frisén \& Wängqvist, 2011; McLean, Syed, Yoder, \& Greenhoot, 2016; Syed \& McLean, 2015). In this paper, we set a course to remedy this imbalance by proposing a model to guide the study of identity content. Infusing identity development research with a full consideration of identity content will not only enhance our understanding of identity processes, but will also facilitate a more contextualized understanding of identity development, a perspective that has been lacking in the field.

\section{The Case for Considering Identity Content}

At the outset we acknowledge that there is attention to the content of identity in literatures across many disciplines. Similar and related constructs such as self-concept, personality, values, and self-esteem, have been studied extensively. However, this transdisciplinary research has not offered a comprehensive model of identity content, and this research is not explicitly developmental. We make the case in this paper that 1) a developmental lens towards content is critical because content is not developmentally invariant, and 2) a comprehensive model of content is needed that includes the most distal components of identity (i.e., culture) down to the most proximal (i.e., everyday experiences). A comprehensive model 
must not only be horizontally broad (covering a range of content within a given level), but must also be deep (covering content at multiple levels). We now turn our attention to the discussion of identity development, the literature in which we situate our arguments.

Much of the research on identity has focused on antecedents and outcomes of various forms of identity processes (e.g., Berzonsky et al., 2013; McLean \& Pratt, 2006; Meeus, 2011; Schwartz et al., 2013; Syed \& Azmitia, 2010; Waterman, 2015). Historically in developmental psychology, the most prominent identity processes were those that constitute the identity status model (Marcia, 1966): the degree to which individuals engage in exploration of various identity alternatives and their feelings of commitment to a particular identity structure. Contemporary research has expanded these two identity processes into more specific forms, such as exploration in breadth, exploration in depth, reconsideration of commitment, and identification with commitment (for a review see Crocetti \& Meeus, 2015). Identity styles, which are closely related to the identity status model, focus on social-cognitive processes that signal how individuals approach the identity task (e.g., normative, informational; Berzonsky et al., 2013). Narrative identity research emphasizes the process of autobiographical reasoning, which is how individuals reason about their past experiences and connect them to their current identities. Specific forms of autobiographical reasoning include meaning-making (McLean, 2015), selfevent connections (Pasupathi, Mansour, \& Brubaker, 2007), and exploratory processing (Pals, 2006), among others.

Based on assessment of these processes individuals are conceptualized as more or less mature in their identity development (e.g., achieved, diffused, reporting more or less meaning), conceptualizations that represent a snapshot of identity at a given point in time. A large, rich literature documents the important psychosocial outcomes that are linked to such processes (e.g., 
Adler Lodi-Smith, Phlippe, \& Houle, 2015; Rivas-Drake et al., 2104; Tsang, Hui, \& Law, 2013).

Further, these processes have been applied to identity conceptualized very broadly - that is without reference to specific components of identity (e.g., Luyckx, Schwartz, Rassart, \& Klimstra, 2016), and to various domains of identity specifically (e.g., sexual identity or ethnic/racial identity; Toomey, Anhalt, \& Shramko, 2016; Yap et al., 2016). And within specific studies, authors have addressed contextual factors, such as family, school, or neighborhood influences on specific aspects of identity development (e.g., McLean, 2015; Rivas-Drake \& Witherspoon, 2013). Others have addressed social and contextual issues, such as discrimination or inequity (e.g., Jones \& Galliher, 2015; Richardson et al., 2015). In sum, identity processes have been quite fruitfully studied, both generally and within certain identity content domains.

The proliferation of research on identity processes across aspects of identity might lead some to conclude that the study of identity process and content are independent, and that one need not think about the other in studying either of them. We argue that the study of identity development is strengthened by an integrative approach that emphasizes both process and content (McLean, Syed, \& Shucard, 2016; Syed \& McLean, 2015). Yip, Seaton, and Sellers (2006) provided a notable recent example of research that integrates the study of process with specific aspects of identity content. In a sample of 940 African American adolescents, emerging adults, and adults, they assessed ethnic identity status (i.e., diffused, foreclosed, moratorium, or achieved) in relation to ethnic identity content (e.g., racial centrality, racial identity ideology). Achieved individuals, those high in both exploration and commitment, reported the highest levels of racial centrality and felt the most positively about their African American heritage. In contrast, diffused individuals, those low on both exploration and commitment, reported the lowest scores on both racial centrality and positivity about their race. We applaud efforts, such as 
this, which seek to consider identity development processes with a clear focus on content. It is our hope that a comprehensive framework for studying content will provide an organizing structure for consolidating such literature.

In part, the prevailing patterns in identity research may stem from the major challenge of studying content. Specifically, focusing on manifestations of identity content largely leads to a mass of descriptive information. Further, identity content can be highly variable and idiosyncratic, and existing taxonomies are largely limited to summaries of types of identity domains (e.g., Balistreri, Busch-Rossnagel, \& Geisinger, 1995). The collection of descriptive results limits the abilities of researchers to answer many of the questions that psychologists seek to answer regarding generalized, universal processes. To consider identity content requires a willingness to develop more contingent models that defy tidy developmental stories.

Despite these challenges, we argue that the mass of descriptive information is absolutely critical for building knowledge. Decontexualizing identity, or making it content-free, provides for neater studies but false comfort. First, it is incomplete to examine a process of development without knowing what is developing. Second, we need to understand what areas of people's lives matter when defining themselves. In doing so, we can understand the full picture of a person's identity - an empirical necessity - but we can also see where, or on what contents, people get stuck - an ethical necessity, particularly for aiding interventions. Thus, we argue that the study of identity content enriches, rather than supplants, the study of identity processes. And, more broadly, it is insufficient to understand only the how, and not the what, of development.

\section{Introducing a Multilevel Model of Identity Content in Context}

A major barrier to studying identity content is that the field of identity research lacks a comprehensive model that first clearly and comprehensively defines identity content, or a 
framework for understanding how multiple forms of identity content interact with each other. The existing literature is rich with examples of identity explorations within specific domains or aspects of identity content, but rarely are multiple contents examined or the interactions between them (see Dehlin, Galliher, Bradshaw, \& Crowell, 2015; Syed, Azmitia, \& Cooper, 2011 for exceptions). The extant literature also includes quite varied definitions of identity content, ranging from broad cultural-ideological belief systems or values to small-scale behavioral manifestations of identity. Theorists and researchers have tackled the complexity of identity development related to many aspects or domains of identity (as an example of this type of sophisticated theorizing, see Verkuyten's (2016) analysis of ethnic identity situated within social context). All of these approaches provide valuable insights into conceptualizations of content, but they also signal the depth and richness of the construct of identity content that cannot be captured with a simplistic model. Thus, rather than a singular definition of identity content, we determined that a more useful approach is a multi-level system that integrates across different levels of content. Our thinking was inspired by Bronfenbrenner's (1979) ecological systems theory, although as described subsequently, our model departs from that theory in important ways. The multilevel model of identity content in context that we propose consists of four interrelated levels of analysis in which to capture identity content, and provides a framework for understanding the existing identity development literature and for guiding future research agendas (see Figure 1): culture, capturing the historical, political, and structural factors in a society; social roles, pertaining to the relational contexts in which identities are developed and negotiated; domains, representing the various life spaces that individuals feel are central to who they are; and everyday experiences, capturing the day-to-day thoughts, feelings, and actions associated with individuals' identities. 
At the broadest level of influence, identity develops within cultural and historical contexts, as individuals respond to cultural messages about what constitutes a "good life," which goals and aspirations are appropriate, and the limits on ways one can define the self. These larger cultural influences frame identity development experiences at the more proximal level, as individuals fulfill the second level of analysis, social roles, and relate with important others in their more immediate contexts. Even more proximally, each individual negotiates multiple aspects of identity within particular domains, the third level, and in the contexts of their everyday experiences, the fourth level, all with the challenge of creating a sense of self that feels coherent and whole both across and within these levels (Klein, Spears, \& Reicher, 2007). Some authors describe this negotiation as the task of integrating or managing multiple identities (e.g., Curtin, Kende, \& Kende, 2016; Kang \& Bodenhausen, 2015); however, in this model, we will use the term "identity" to capture the integrated, contextualized, holistic sense of self, which is comprised of numerous aspects of self.

This model, which moves from distal to proximal, is clearly inspired by the concentric circles of Bronfenbrenner's (1979) ecological systems theory. However, it does not map directly for two primary reasons. First, rather than sources of "influence" on the developing child, the four levels of content are seen as embedded directly within individuals' developing identities. In other words, we take the perspective that culture is 'alive' in individuals who are actively engaged with cultural practices, and those individuals can be seen as vehicles for understanding the larger culture. Second, a major point of departure from Bronfenbrenner or other similar models is that the current model explicitly takes notions of power and privilege into account, particularly at the level of culture. In elaborating this component of our model we draw from theories on intersectionality (Crenshaw, 1991), which emphasize the unique experiences of 
individuals who are positioned at the intersections of multiple marginalized identity domains. Intersectional theorists note that privilege exists in many forms and possessing multiple marginalized identities leads to unique experiences of marginalization, encompassing ideas of both additive stress as well as resilience (Purdie-Vaughn \& Eibach, 2008). Most central to the current argument, however, is that an intersectional approach addresses the inherent need to be seen as a whole person, rather than divided into different aspects or selves. This is an advantageous lens to bring to the study of identity content vis-à-vis the goal to understand identity development of the "whole person."

Finally, in articulating this framework for studying content we do not neglect identity processes. We do, however, draw attention to some different processes that have been less wellrepresented in the literature. The typical approach is to assess processes associated with a particular identity domain (e.g., exploration, commitment) or generalized sense of self (e.g., autobiographical reasoning). Rather than these domain-specific processes, we emphasize processes that capture the dynamics of identity content. That is, rather than processes tied to a specific domain, we highlight processes pertaining to the system of contents. This conceptualization of process renders the consideration of both process and content necessary, and therefore enhances our conceptualization of identity content.

The processes we highlight throughout the model follow from the Eriksonian concept of identity configurations (Hammack, Thompson, \& Pilecki, 2009; Schachter, 2004; Syed, 2010). Identity configurations correspond to the ways in which individuals arrange, manage, or configure, the different aspects of their lives. This conceptualization gives rise to three specific processes for understanding identity content within a multi-level system: conflict, compartmentalization, and coherence. Many individuals experience intense conflict as different 
aspects of their identities appear "at odds." Some people may remain in this conflicted state, obviously a source of great distress, and some may compartmentalize various components of identity to potentially reduce distress. Identity coherence has often been described as an optimal identity development outcome, characterized as a clear, articulate, and firmly embraced sense of self, which we argue includes a sense of harmony among identity contents, at various levels. We will highlight each of these three processes in our discussion of the model.

As we move to discuss our framework for capturing content, we do caution readers not to move too quickly beyond the study of description to the study of the processes of conflict, compartmentalization, and coherence, because we still have much work to do as a field to define content before we can fully understand the processes used to negotiate content. With that caveat in mind, we now turn to the description our framework for capturing content (see also Figure 1).

\section{Level 1: Cultural and Historical Contexts of Identity Development}

We contend that it is necessary to situate the development of identity content within its historical and cultural contexts. Particularly relevant is an understanding of systems of power and privilege that elevate some forms of identity over others in terms of access to status, resources, and social capital (e.g., McIntosh, 2012; Rothenberg, 2016). Identity labels can often be framed in complementary roles as "marginalized" or "dominant." From an intersectional perspective, the forces of discrimination and prejudice are not only relevant for the identity experiences of individuals with marginalized identities, but also for those from relatively dominant identities, as their relative immunity from oppression is a crucial aspect of their identities.

Indeed, those holding dominant identities benefit from systems of privilege that confer authority, wealth, and status inequitably. For example, in contemporary U.S. society, systems of privilege benefit those who identify as male, White, heterosexual, able bodied, English speaking, 
well-educated, Christian, and affluent. Those who hold those identities have the luxury of never having to consider their dominant identities, and often report very low levels of identity awareness (e.g., Juan, Syed, \& Azmitia, 2016; Rivas-Drake et al., 2014). In other words, holding the dominant identity status can render identity invisible (Fivush, 2004, 2010), as those with privilege conceive of themselves as simply "normal." Ironically, those who hold privileged identities may very well acknowledge the existence of oppression for those who hold marginalized identities, but still struggle greatly to acknowledge their own privilege (the flipside of oppression; McIntosh, 2012), with consequent societal costs in terms of changing the systems and structures of oppression. For many individuals, identity development involves the negotiation of aspects of self that experience privilege and aspects that do not experience privilege, with clear societal level messages about which forms of identity are valued over others.

The idea of master narratives is a useful concept for understanding the way in which privilege manifests in the interplay between personal identity and culture. Using this perspective, scholars have argued that cultural norms and expectations are communicated in ubiquitous cultural narratives about how a life should be lived, as well as in the creation and maintenance of narratives about shared historical events, and shared structures for telling personal stories (Hammack, 2008; McLean \& Syed, 2015). Those whose personal narratives align with these master narratives, are the privileged and 'normal' referenced above. Those whose personal narratives deviate from these master narratives are in a position of marginalization, and are also tasked with more identity work to explain one's deviation and find a place to belong, or an alternative narrative with which to align. Thus, for those with marginalized identity labels, these aspects of self may be experienced as more salient because of their apparent deviation from 
"normal," and individuals must then strive to achieve a positive sense of self in the context of discrimination, invisibility, and invalidation (e.g., Bombay, Matheson, \& Anisman, 2010; Sanders Thompson, 1999). Alternatively, some may be motivated to compartmentalize, or even minimize, identities that are linked to lower status, instead emphasizing aspects of identity that align with master narratives, or are linked to greater acceptance from the culture at large.

These processes are both historically and culturally contextualized, as are broad systems of power and privilege. The theory of intersectionality was conceived as an interpretive framework to understand interlocking systems of oppression. As it has been increasingly applied within the individual-focused field of psychology (Bowleg, 2008; Cole, 2009; Rosenthal, 2016), there are additional issues that must be taken into account (Juan et al., 2016; Syed, 2010). Most central for the present model is the slippery slope of equating intersectionality with the study of multiple dimensions of identity (see Syed \& McLean, 2015). The examination of how individuals develop and manage multiple identity domains is necessary but not sufficient to be an intersectional analysis. Rather, intersectionality, by definition, must involve an analysis of power, inequality, and injustice as a central concern. An emerging literature in this respect is certainly developing in psychology, expanding the concept in new and interesting ways.

While theory and research related to intersectional identity has addressed the experiences of those who possess multiple marginalized identities, other forms of identity negotiation exist. Identity strain emerges at the intersections of marginalized and mainstream identity domains, and between other aspects of identity. A number of authors have discussed the process of developing a positive, coherent same-sex attracted identity, within the context of a deeply held, nonaffirming religious identity (e.g., Dahl \& Galliher, 2012; Dehlin et al., 2015). Others have written about the challenges of women in male-dominated career contexts (e.g., engineering, Hatmaker, 
2013). Given the complexity of the identity structure for any individual, an ongoing process of negotiation must be engaged across the lifespan, in order to negotiate identity across domains.

Socialization processes are a critical space for communicating these cultural messages. Socialization practices in families and communities communicate to children, as well as adults across the lifespan, which aspects of identity are desired and which are to be avoided or hidden. These cultural cues, often coming in the form of master narratives, shape an identity salience hierarchy, in which individuals determine which aspects of their identities are most valued by others, and ultimately determine which aspects of identity are experienced subjectively as most primary. Thus, examining identity content at this level not only tells us more about the individual, but also about the culture in which the individual lives (McLean \& Syed, 2015). This issue is central to the increasing calls for psychologists to move beyond the study of the relatively privileged (e.g., WEIRD samples; Henrich, Heine \& Norenzayan, 2010), to encompass the whole of society to understand all of the individuals that make up that society, as well as the society itself.

One recent example of the interplay of culture, history, and politics in identity development comes from Houkamau's (2010) study of the life stories of Māori women in New Zealand spanning generational cohorts born from the 1930s through the 1980s. Women born prior to the 1950s centered their identity descriptions around traditional spiritual and collectivistic cultural values, reflecting their early experiences embedded in traditional Māori communities. Those born in the 1960s described a sense of alienation from their Māori identity, as migration to urban centers and the forces of discrimination led families to distance themselves from their Māori ancestry. Finally, the identity related stories of women born after the 1970s reflected their political engagement, awareness of social injustice, and emphasis on Māori civil 
rights. Importantly these differences in generations are not only tied to beliefs and practices about different groups in society, but also to structural changes, such as migratory pathways and the legal changes regarding access to resources and legislation regarding land rights. Hammack (e.g., Cohler \& Hammack, 2006) has made similar observations regarding the making of gay identities, situating his examination in the context of master narratives, showing that that the "coming out" process is generationally contoured and linked to societal changes in the meaning of gay identities (see also Weststrate \& McLean, 2010). The rich literature on identity conflicts among LGBTQ individuals in conservative religious contexts also provides a powerful example of the strategies used to negotiate such conflict (e.g., Dahl \& Galliher, 2012; Dehlin et al., 2015), as individuals often feel as if they have to choose one component of identity over another, or have to compartmentalize identity contents into different times and spaces within their lives.

\section{Level 2: Social Roles}

Relational and social identity is an aspect of the self that develops in the context of relationship roles (Tajfel, 1978), and that continues to shape one's overall sense of self. There are an unlimited number of social roles one may engage, such as brother, colleague, mentor, mother, or spouse. These roles are connected to Level 1 in that they are both defined by the culture (e.g., in China the role of sibling may be irrelevant for some generations), and given meaning by the culture (e.g., the role of spouse may vary greatly in cultures that have arranged versus chosen marriages). Social roles become integrated into the overall sense of self, becoming identity labels themselves, and as noted below, social roles intersect with domains of identity that have historically been characterized as "personal identity" (Level 3).

The level of social roles captures several distinct components of identity content compared to other levels. First, social roles are explicitly defined by their relationship to others. 
That is, there is a required connection beyond the self. Identity domains (e.g., politics, religion), which are described below can, in theory, be entirely self-chosen and self-determined. But this is not the case for roles, which are defined at least in part by other people. Second, we also argue that social roles constitute their own level of analysis because we want to draw attention to a component of identity that has been relatively ignored in the developmental literature, perhaps because many of these roles (e.g., worker, parent, spouse) emerge in adulthood, a stage of relative neglect within the field of developmental psychology.

Scholars have examined the relevance of specific social roles both individually and together. On the individual level, for example, Adamsons and Pasley (2016) explored the salience and centrality of the role of father as couples transitioned to parenting, finding that greater father role centrality linked to higher levels of father involvement in subsequent years. However, a more ecologically valid approach would be to examine roles in interaction with one another, and in interaction with aspects of the self at other levels of our model. For example, Kanji and Cahusac (2015) explored the identity transition experiences of 24 working women over the course of their pregnancies. Women initially experienced challenges in embracing identities as mothers within their work contexts, with almost all women hiding their pregnancies through the first trimester and worrying about the impact of motherhood on their professional identities. Interestingly, women in female dominated work settings who reported the availability of role models for the transition to motherhood described increasingly strong and optimistic identities as mothers over the course of pregnancy. Those who lacked female role models in male-dominated professions continued to feel stress about integrating their identities as mothers with their professional identities, and felt forced to choose between their maternal and professional selves. This study nicely exemplifies the processes of conflict, 
compartmentalization, and coherence (for some) within two specific contents. Further, these authors captured complex negotiations between a developing role-based aspect of identity (motherhood), and an existing aspect of personal identity (professional identity). Again, we argue that not knowing what contents are in conflict is highly problematic. Understanding that these two contents may be particularly ripe for conflict, perhaps especially for women, guides theorizing about women's identity development, as well as intervention efforts in the workplace.

Finally, group memberships and social self-representations represent critical aspects of the self that may bridge the gap between the relational level of identity content and domains of personal identity. A large body of literature that examines political identities linking individuals to specific parties or political movements (Kidder, 2016), religious identities related to membership in a specific religious organization (Hichy, Gerges, Platania, \& Santisi, 2015), occupational identity work in the context of specific job categories (van Vuuren, Teurlings, \& Bohlmeijer, 2012), and countless other unique group affiliations. Those components of identity are defined by relationships with a particular community, but also involve a subjective, internal process of meaning making that occurs at a more individual, personal level.

\section{Level 3: Domains of Identity Content}

Despite the focus in much identity research on broad and general identity processes related to a general or overarching identity (e.g., Bosch \& Card, 2012; Crocetti et al., 2015), a large body of literature also acknowledges unique identity development experiences across domains of identity within individuals. Individuals develop a sense of self related to gender, ethnicity, religion, occupation, and romance among many other aspects of identity. The processes of identity development are certainly not expected to remain parallel across all domains of identity. Thus, an individual may have engaged in a great deal of identity work 
regarding ethnicity or religion, but not with regard to occupational or gender identity (McLean et al., 2016; McLean, Shucard, \& Syed, 2016). Therefore, in order to properly understand process, we must also consider the relevant contents. This is the case not only between domains, but also within domains.

This is the level for which there exists a working taxonomy, to some extent. Within the identity status literature, researchers have defined eight content domains (e.g., Balistreri et al., 1995), in which individuals may define themselves in terms of ideological (religion, occupation, politics, values), and interpersonal concerns (romance, friendship, family, gender roles). Recent research has shown that these are relevant domains to emerging adults, though they do not constitute a comprehensive taxonomy, and individuals vary in which are more salient or important to them (McLean, Syed, \& Shucard, 2016). Thus, a major step forward would be to build on this work by defining domain contents, determining their presence and unique manifestation across groups within a society (e.g., men versus women) and developmental stages, and their salience across groups and developmental stages. In other words, we do not expect this to be an invariant taxonomy, but research will need to investigate this claim.

Domain interactions. As we have mentioned, domains of identity do not simply develop in parallel, but interact in complex ways. For example, one's identity as a woman is different depending on whether she is a lesbian or heterosexual. As is clear in the current political climate in the U. S. in 2016, gender and political identity are intertwined, as women must explain their allegiance to politicians who have denigrated women, or their lack of allegiance to a female politician. Given the complexity of the identity structure for any individual, an ongoing process of negotiation must be engaged across the lifespan, in order to negotiate identity across these levels of analysis. 
Empirically, however, we also see the difficulty involved in articulating overlapping content domains and roles. When asking individuals to generally describe personally defining narratives, the majority of emerging adult participants $(\sim 70 \%)$ talked about one domain only, such as family or values (McLean, Syed, \& Shucard, 2016). A smaller proportion discussed the intersection of two domains, and very few went beyond two domain contents, perhaps due to the challenge of conceptualizing such interactions (Azmitia, Syed, \& Radmacher, 2013). Thus, at least in emerging adulthood, the majority of individuals seem to articulate one content at a time.

In another study, in which we asked for identity-defining narratives specific to content domains (Authors, 2016), some contents were more likely to 'spill' across narratives. For example, no matter what content domain we solicited (e.g., dating, religion, values), family was likely to appear. In general, interpersonal domains were present across various contents, suggesting that this content domain is closely connected to other domains. Other domains were less likely to appear with other contents, and these were largely the ideological contents, including gender. Of course, these patterns may change with development, a question for future research; once the content patterns have been specified, examining processes of change and stability is the obvious next step.

\section{Level 4: Enacting Identity in Everyday Interactions}

Levels one through three of this model discuss identity content in relatively abstract, meta-level terms. However, a critical component of the study of identity content is examination of the lived experiences of the individuals we are seeking to understand. This level of analysis is not amenable to the self-report survey methodology that is dominant in the identity development literature. Accordingly, this level is less well represented and less well understood than the other levels. Its value, as will be discussed, is twofold. First, analyses of micro-content provide a 
phenomenological perspective to identity — what daily life "looks like" for individuals who represent particular identity structures. Second, micro-content experiences are sites in which analyses of the previous levels can be understood, as manifest in specific behavioral episodes. That is, the importance of culture, relations, and domains can potentially be revealed through the micro-content. There are two methodologies that have addressed this level particularly well: narrative studies and experience sampling studies. We will describe each, in turn, but first we offer the following narrative from one of our studies (Syed \& Azmitia, 2010) as an example of this kind of work. This narrative came from a Mexican-heritage college student, responding to a prompt for an experience when she became aware of her ethnicity.

It was in my first Intro to Latin American and Latino Studies class. We were having a debate in class or something and they were talking about bilingual education and like I didn't know whether to be for or against it. It was kind of hard. It was kind of, well I think what affected me more was to hear people's ignorance like in my own group. That people that were against me were like well you know you're in the United States so you have to speak English. And I was kind of like well who are you to say that they came here willingly or you know. It just goes to show me how much I do like see, like there are those people that like say yeah, "I'm Mexican American but you know that's not what makes me," you know, but me, that is what makes me, I think. Like that's something that I cannot get rid of, you know. (p. 215)

Narrative studies involve prompting respondents to recall and describe memories of specific experiences from their past (McAdams, 2001), as in this prompt above. The majority of this research has focused on the processes of narration and what they reveal about identity (McAdams \& McLean, 2013). However, the approach is particularly applicable for understanding content as everyday experiences, as narrative analysis facilitates an understanding of the lived experiences that are relevant to specific content domains. For example, in the ethnic identity literature, narrative studies provide insight into how ethnicity is activated and managed in peoples' everyday lives (see Syed, 2015, for a review). Moreover, the types of experiences narrated reliably align with individuals' developmental processes. Those who are more strongly 
identified with their ethnicity are more likely to tell stories about either discrimination experiences or feeling a close connection to their culture (Syed, 2015), and those who are less strongly identified tell stories like the one above, classified as an awareness of difference story. Collecting memories of specific moments in people's lives provides a wealth of information about not only the general type of experiences that are relevant to particular identity domains, but also where they were when the event happened, who they were with, and how they reacted. These details provide endless opportunities for a fully contextualized study of identity—content, process, and the interaction of the two.

The analysis of specific experiences not only adds to the elaboration of the aspects of self we seek to understand, but also helps us to understand how the three other levels intersect within one person. For example, in the narrative above see how cultural expectations about what it means to be an American (e.g., speaking English) are not only communicated in the very local classroom context, but also intersect with societal structures (e.g., the education system). Similarly, McLean, Shucard, and Syed (2016) found that individuals differed in the way in which they negotiated cultural expectations about being male or female in their life experiences, with some individuals reporting specific experiences about conforming to traditional cultural expectations about being male or female (e.g., men playing more assertive roles in dating), others focused on specific experiences of discrimination, and yet others reporting experiences of 'sameness' - with examples emphasizing how women can do what men can do and vice-versa. Thus, within one content domain - gender - individuals have exposure to different cultural experiences that create variation in the manifestation of content.

When individuals discuss their experiences within a content domain we can see how some domains connect to others. For example, the domain of family seems to be relevant to 
many other domains, such as values and religion (McLean et al., 2016; McLean, Syed, \& Shucard, 2016). These experiences also reveal how social roles are embedded within domains. For example, in narratives about gender some individuals discuss their roles in romantic relationships as indicative of their understanding of themselves as male or female (McLean, Shucard, \& Syed, 2016). Finally, these everyday experiences are implicitly, and sometimes explicitly, embedded in the level of culture. One can 'read' these personal narratives for the larger cultural expectations, beliefs, and structures, with which individuals are negotiating (McLean \& Syed, 2015). In some ways, examining this micro-context of identity is a way to elaborate, and specify, the other levels of this model.

In contrast to narrative studies that focus on individuals' reconstruction of their past experiences, the experience sampling method (ESM) involves studying psychological phenomena in vivo (Hektner, Schmidt, \& Csikszentmihalyi, 2007; Thomas \& Azmitia, 2016). This is accomplished by using electronic devices (e.g., beeper, cell phone) to prompt respondents to complete a brief response battery at various moments throughout the day for a specified period of time (often two weeks). Despite its promise for contributing to understanding of both content and process, the ESM method has been used quite infrequently in the identity literature. ${ }^{1}$ One notable exception is Yip's (2005) study of situational salience in ethnic identity among Chinese American college students. Content-relevant questions included the ethnic composition of their current setting, whether they were with family, and the predominant language being used. Among many other findings, results indicated that all three of those contextual cues were associated with greater situational salience of their ethnicities. Thus, ESM studies can contribute to understanding the moments in individuals' lives when particular identity contents become

\footnotetext{
${ }^{1}$ The related "daily diary" method has been used more often (Klimstra, et al., 2010; Schwartz et al., 2011; Yip \& Fuligni, 2002). However, this method involves participants completing a brief survey once per day at their convenience, rather than random points throughout the day as they are leading their lives.
} 
activated and, by extension, how they psychologically manage that activation. Such studies would be a welcome contribution to the literature.

\section{Implications of the Multilevel Model for Identity Research}

We have highlighted complexities within and across the levels of this model, which may frame future empirical investigations. These complexities are not meant to frustrate researchers seeking tidier designs, but to show these levels of the model can fit together, and to show the necessity of embracing these complexities. If researchers target only one level of identity development, without considering the broader contexts, this will limit our understanding of identity development. For example, examining the domain of occupation, without considering how it intersects with the domain of gender and the cultural expectations about that intersection, would constitute an incomplete analysis.

\section{Conflict, Compartmentalization, Coherence Redux}

Having now defined the four levels at which one may see identity content, we return to a more focused discussion of process. When taking a sole focus on process, researchers have found that those with identity conflict (Szymanski \& Carr, 2008), and in some cases those with compartmentalized identities (Showers \& Ziegler-Hill, 2007), experience less positive adjustment than those who maintain a more coherent identity. Yet, in large part, these prior studies have not specified what the conflict, compartmentalization, or coherence, is about. These decontextualized, content-free analyses fall short of our responsibility to understand identity development in full, and in ways that lay foundations for translational or interventional work. For example, as younger people learn about the expectations of others, and become attuned to them across adolescence, the location of conflict may lie between personally salient domains and cultural expectations (e.g., Dehlin et al., 2015), perhaps a location of conflict that shifts as 
identity becomes more integrated and individualized in adulthood. Beyond understanding more about identity development, understanding the location of these conflicts should play a major role in interventions to alleviate psychological distress. Thus, these processes of identity development become particularly illuminated once identity content is considered.

There are a number of strategies for examining these processes within content domains. For example, one can experience conflict between the roles that make up the content domain of gender - such as the pull between mother and daughter, when one's parents begin to age. One can also experience conflict between domains, such as the woman who struggles with her religious and political identities. Perhaps the most likely form of identity strain involves the synthesis of domains of content that appear to be "at odds" with one another. For example, the conflict between one's experiences as a black police officer and the cultural expectations of being a black man is a negotiation that bridges ethnic identity and occupational identity. Some may compartmentalize their identity contents so that they do not rub up against each other. Others may find a way to explain their edges of intersection that makes sense. And, of course, these ways of managing conflict will have ramifications for social roles, and for the way that they fit into acceptable cultural definitions of identity.

When thinking about these processes of conflict, compartmentalization, and coherence, there are additional concerns regarding the substance of the identity contents that must be taken into account. Below we provide details for two such concerns: 1) whether the identity is assigned by others and/or society versus self-chosen and 2) if the aspects of identity are visible versus invisible to others. These concerns are particularly relevant in the context of our proposed model, and they involve an intersection of the cultural, relational role, domain, and everyday levels of analysis. 
Assigned versus chosen aspects of identity. We define an assigned identity as one that is relatively stable across time, and difficult, if not impossible to change (e.g., sex, ethnicity, adoption status). For assigned identities, the task is to understand that identity, to make meaning of it. These meanings and understandings can surely change over time, but the identity content is always there. For other aspects of identity, the task is more about constructing or discovering that aspect of self (Grotevant, 1992). For example, one is not born a parent or a teacher. These are aspects of identity that emerge, which are most often chosen, but that still need meaning-making processing. Although both types of identity contents may carry with them a set of expectations about how that identity is to be enacted, whether one has chosen that identity may shape how processes are enacted. Moreover, interactions among multiple domains can potentially involve an interaction between an assigned and a chosen identity. The research in the U.S. on ethnic minorities in science, technology, engineering and math (STEM) fields is a notable example. Students from ethnic minority backgrounds (assigned) in STEM fields (chosen) are underrepresented relative the population and face many barriers to entry and persistence in these fields. Because of this, they oftentimes must engage in identity work around their ethnicity, as their under-representation serves as a context that makes those identities salient (Nasir \& Saxe, 2003; Syed, Azmitia, \& Cooper, 2011).

Identity labels may be assigned based on changing life circumstances, and also necessitate meaning making processes. There may be normative changes, such as the adoption of a parenting identity. There may also be idiosyncratic changes, such as a cancer diagnosis that may precipitate the development of an identity as a "cancer patient" - an identity that may remain relatively stable over time, or may be let go following successful treatment (Cho \& Park, 2015). Identifying such temporal changes will tell us about broad changes in identity concerns between 
persons (such as age-related valuing of occupational or parenting identity), as well as withinperson changes that will help us to understand psychological functioning. For example, youth diagnosed with cancer who endorsed more empowering self-labels experience better psychological adjustment over time (Cho \& Park, 2015). Without accounting for identity content, we are left in the dark about why we see such changes in psychological functioning. Continuing our current example, without accounting for identity contents, we might not know if the change in psychological functioning for cancer patients was change due to successful treatment or to the adoption or letting go of specific aspects of one's identity.

Other aspects of identity are more fluid and explicitly involve either a sense of choice or intention. For example, a young person from a working class background can strive to achieve an education and a high-status career, in order to move away from a working class identity and toward an identity as a "professional." Of course, such an identity transition is embedded within systems of inequity (Level 1), and thus is not without conflict or constraint. Stahl (2016) critically evaluated the relevance of the dominant neoliberal discourse related to academic/economic success and upward social mobility for lower class young men.

Disidentification with "middle class," with corollary low academic achievement and antisocial behavior, is viewed as a function of complex blends of cultural messaging and disparities in opportunity. His sample of 24 lower socioeconomic status boys in London emphasized a sense of loyalty to self, and a desire to feel authentic and genuine in their school related interactions. Primarily, they viewed this authenticity as not being "showy," just being "average," and being the same at school as they are outside of school. Young men were very concerned about being seen as pretentious or self-important, and resisted the achievement oriented, competitive narrative associated with academic and economic advancement. These examples show how 
Levels 1, 2, and 3 interact in complex ways, underscoring our claim that there may not be tidy models for examining identity content, but that by examining content we better understand identity development.

Finally, chosen aspects of identity can come in the form of acquired characteristics, leisure activities, group affiliations, and other unique aspects of the self. The unique aspects of identity that can become incorporated into the overall identity are limitless, as can be seen through the breadth and variability in the identity literature - athletic identity (Martin \& Horn, 2013), sports fan identity (Koch \& Wann, 2014), academic teacher identity (McNaughton \& Billot, 2016), “gamer” identity (De Grove, Courtois, \& Van Looy, 2015), and many others. Although some are born San Francisco Giants fans, unique self-labels largely evolve throughout development, so an individual may identify at various times across the lifespan as a rock climber, student, or scientist.

Visible vs. invisible aspects of identity. Individuals are members of social categories based on characteristics that are either visible to others or not visible to others. Some aspects of identity will only be known to others if a person chooses to disclose them. Ragins, Singh, and Cornwell (2007) explored decisions about disclosure of sexual orientation in a large, U.S. sample of lesbian, gay, and bisexual employees in their workplaces. Concealment of non-heterosexual orientation and fear of disclosure were linked to a number of negative work-related and personal outcomes, and the authors highlighted the strain associated with managing stigmatized aspects of identity within discriminatory and sometimes hostile contexts.

Some aspects of identity are made visible through verbal and non-verbal behavior or through display of artifacts, such as religious icons, clothing choices, or other signs and markers. In contrast to Ragins et al.'s (2007) exploration of sexual identity concealment, Morgan and 
Davis-Delano (2016) described explicit and often intentional behaviors engaged by heterosexual individuals in order to "mark" or display their heterosexual identity (e.g., gender conforming behaviors, displaying other-sex sexual interest). These marking behaviors are situated within the context of power and privilege, as participants acknowledged that ensuring that they are perceived as heterosexual is a strategy for avoiding the prejudice and discrimination associated with a sexual minority identity. Further, heterosexual marking was conflated with gender conformity, and for men, heterosexual marking served to distance themselves from both a stigmatized non-heterosexual sexual orientation and devalued femininity.

Other aspects of identity are highly visible (Alcoff, 2006), such as biological sex or ethnicity. For such aspects of identity, development will be a negotiation of subjective identity considerations and assumptions or ascriptions placed upon the individual from the outside. For example, if a person is born with female genitalia, assumptions and expectations will be placed upon that person regarding the expression of a gender identity. The assumptions and expectations include stereotypes about what women can or cannot do, discriminatory or prejudiced attitudes, and value judgments about adherence to gender roles. Thus, it is clear that when we attend to content, we begin to understand cultural context much more clearly, and this becomes especially relevant when looking at issues of privilege, power, and oppression.

In sum, conceptualizing identity negotiations through the lens of our multilevel model confirms that if we do not know where identity conflicts lie - what spaces they take up - we do not know if interventions need to focus on individualized work, relational work, cultural change, or some combination of the three. For example, to continue to address identity conflict that rests upon the cultural constraints of being a black man by targeting the psychological experiences of that man, without attention to the structure in which that man lives, will lead to continued 
personal distress through generations, not to mention the societal costs associated with the distress. If we continue to hold up those who experience identity coherence as models of successful identity development, without recognizing the constraints placed on various contents for some, we unnecessarily blame others for their failure to make sense of the senseless.

\section{Concluding Remarks and Recommendations}

We offer here some ideas for how this framework can be used to both better capture the phenomenon of identity development, and to push theory and basic research, as well as the application of that research. First, our model puts culture and context at the forefront of studying identity development. Not attending to culture or context in the study of a concept that is so culturally and contextually situated is deficient. Second, we need to continue the expansion of descriptive scientific work that captures complex, interacting, and sometimes idiosyncratic aspects of identity content. One of the major limitations with existing approaches is that we may

not be capturing all, or even the most important, processes if we do not know what we are talking about first. Indeed, describing a phenomenon accurately and fully may lead to different ways of conceptualizing process - we may be missing something. Third, we need to understand both normative content, as well as more idiosyncratic content. With which contents should we see most people, in a given culture, at a given developmental stage, wrestling? And which contents will be particularly important for which people? What contents are more variable in their importance, and for whom? Finally, related to the third issue, if research on identity development is to be adequately applied to real-world interventions, a lack of attention to content may limit the usefulness of interventions. For example, in the Yip et al. study described earlier, if researchers knew only that ethnic identity was diffused, how would they know where to 
intervene? Once the knowledge that racial identity salience as a crucial variable was illuminated, interventions may be constructed to target that specific aspect of content.

The study of identity development is not easy. Identity pertains to individual lives, to relationships, to domains of engagement, and to the culture at large; it can be idiosyncratic, and it is not culturally invariant. The study of identity requires multiple methods and theoretical foundations to fully capture the phenomenon. Indeed, there are many 'flavors' of identity development research. Our task here was not to detail all of the approaches, but to make an argument for what all of the approaches need to consider. We encourage other scholars to embrace this complexity, engaging multiple levels of analysis from the historical and cultural context to the everyday experiences that those larger contexts infuse, and in which those larger contexts are given life. We argue for embracing Erikson's emphasis on integration and wholeness - within a person, between persons, and within a larger cultural context - in order to understand the developmental processes of identity development, and its content. 


\section{References}

Adamsons, K., \& Pasley, K. (2016). Parents' fathering identity standards and later father involvement. Journal of Family Issues, 37, 221-244. doi:10.1177/0192513X13514407

Adler, J.M., Lodi-Smith, J., Phlippe, F.L., \& Houle, I. (2015). The incremental validity of narrative identity in predicting well-being: A review of the field and recommendations for the future. Personality and Social Psychology Review, 20, 142-175.

Alcoff, L. M. (2006). Visible identities: Race, gender, and the self. New York: Oxford, Archer, S. L. (1985). Career and/or family: The identity process for adolescent girls. Youth \& Society, 16, 289-314.

Archer, S. L. (1985). Identity and the choice of social roles. New Directions for Child Development, 3079-99. doi:10.1002/cd.23219853007

Azmitia, M., Syed, M., and Radmacher, K. (2008). On the intersection of personal and social identities: Introduction and evidence from a longitudinal study of emerging adults. In M. Azmitia, M. Syed, \& K. Radmacher (Eds.), The intersections of personal and social identities: New Directions for Child and Adolescent Development, 120, 1-16. New York, NY: John Wiley and Sons.

Balistreri, E., Busch-Rossnagel, N. A., \& Geisinger, K. F. (1995). Development and preliminary validation of the Ego Identity Process Questionnaire. Journal of Adolescence, 18(2), 179192.

Berzonsky, M. D., Soenens, B., Luyckx, K., Smits, I., Papini, D. R., \& Goossens, L. (2013). Development and validation of the revised Identity Style Inventory (ISI-5): Factor structure, reliability, and validity. Psychological Assessment, 25, 893-904. 
Bosch, L. A., \& Card, N. A. (2012). A meta-analytic review of Berzonsky's Identity Style Inventory (ISI). Journal of Adolescence, 35, 333-343. doi:10.1016/j.adolescence.2011.08.007

Bombay, A., Matheson, K., \& Anisman, H. (2010). Decomposing identity: Differential relationships between several aspects of ethnic identity and the negative effects of perceived discrimination among First Nations adults in Canada. Cultural Diversity and Ethnic Minority Psychology, 16, 507-516. doi:10.1037/a0021373

Bowleg, L. (2008). When Black+ lesbian + woman $\neq$ Black lesbian woman: The methodological challenges of qualitative and quantitative intersectionality research. Sex Roles, 59(5-6), $312-325$.

Bronfenbrenner, U. (1979). The ecology of human development. Cambridge, MA: Harvard University Press.

Cho, D., \& Park, C. L. (2015). Cancer-related identities in people diagnosed during late adolescence and young adulthood. British Journal of Health Psychology, 20, 594-612. doi:10.1111/bjhp.12110.

Cohler, B. J., \& Hammack, P. L. (2006). Making a gay identity: Life story and the construction of a coherent self. In D. P McAdams, R. Josselson, \& A. Lieblich (Eds.), Identity and story: Creating self in narrative, (pp. 151-172). Washington, DC: American Psychological Association.

Cole, E. R. (2009). Intersectionality and research in psychology. American Psychologist, 64(3), 170.

Crenshaw, K. (1991). Mapping the margins: Intersectionality, identity politics, and violence against women of color. Stanford Law Review, 43, 1241-1299. 
Crocetti, E., Cieciuch, J., Gao, C., Klimstra, T., Lin, C., Matos, P. M., \& ... Meeus, W. (2015). National and gender measurement invariance of the Utrecht-Management of Identity Commitments Scale (U-MICS): A 10-nation study with university students. Assessment, 22, 753-768. doi:10.1177/1073191115584969

Crocetti, E., \& Meeus, W. (2015). The identity statuses: Strengths of a person-centered approach. In K. C. McLean, M. Syed, K. C. McLean, M. Syed (Eds.), The Oxford handbook of identity development (pp. 97-114). New York, NY, US: Oxford University Press.

Curtin, N., Kende, A., \& Kende, J. (2016). Navigating multiple identities: The simultaneous influence of advantaged and disadvantaged identities on politicization and activism. Journal of Social Issues, 72, 264-285. doi:10.1111/josi.12166

Dahl, A., \& Galliher, R. V. (2012). The interplay of sexual and religious identity development in LGBTQ adolescents and young adults: A qualitative inquiry. Identity: An International Journal of Theory and Research, 12, 217-246. doi:10.1080/15283488.2012.691255

De Grove, F., Courtois, C., \& Van Looy, J. (2015). How to be a gamer! Exploring personal and social indicators of gamer identity. Journal of Computer-Mediated Communication, 20, 346-361. doi:10.1111/jcc4.12114

Dehlin. J. P., Galliher, R. V., Bradshaw. W. S., \& Crowell, K. A. (2015). Navigating sexual and religious identity conflict: A Mormon perspective. Identity: An International Journal of Theory and Research, 15, $1-22$.

Fivush, R. (2004). Voice and silence: A feminist model of autobiographical memory. In J. Lucariello, J. A. Hudson, R. Fivush, \& P.J. Bauer (Eds.), The development of the mediated mind: Sociocultural context and cognitive development (pp. 79 100). Mahwah, NJ: Lawrence Erlbaum Associates Inc. 
Fivush, R. (2010). Speaking silence: The social construction of voice and silence in cultural and autobiographical narratives. Memory, 18, 88-98.

Frisén, A., \& Wängqvist, M. (2011). Emerging adults in Sweden: Identity formation in the light of love, work, and family. Journal of Adolescent Research, 26(2), 200-221.

Grotevant, H. D. (1992). Assigned and chosen identity components: A process perspective on their integration. G. R. Adams, T. P. Gullotta, R. Montemayor (Eds.), Adolescent identity formation (pp. 73-90). Thousand Oaks, CA, US: Sage Publications, Inc.

Hammack, P. L. (2008). Narrative and the cultural psychology of identity. Personality and Social Psychology Review, 12, 222-247. doi:10.1177/1088868308316892

Hammack, P. L., Thompson, E. M., \& Pilecki, A. (2009). Configurations of identity among sexual minority youth: Context, desire, and narrative. Journal of Youth and Adolescence, 38(7), 867-883.

Hatmaker, D. M. (2013). Engineering identity: Gender and professional identity negotiation among women engineers. Gender, Work and Organization, 20, 382-396. doi:10.1111/j.1468-0432.2012.00589.x

Hektner, J. M., Schmidt, J. A., \& Csikszentmihalyi, M. (2007). Experience sampling method: Measuring the quality of everyday life. Sage.

Henrich, J., Heine, S. J., \& Norenzayan, A. (2010). Most people are not WEIRD. Nature, 466(7302), 29-29.

Hichy, Z., Gerges, M. H., Platania, S., \& Santisi, G. (2015). The role of secularism of state on the relationship between Catholic identity, political orientation, and gay rights issues. Journal of Homosexuality, 62, 1359-1373. doi:10.1080/00918369.2015.1060068 
Houkamau, C. A. (2010). Identity construction and reconstruction: The role of socio-historical contexts in shaping Māori women's identity. Social Identities: Journal for the Study of Race, Nation And Culture, 16, 179-196. doi:10.1080/13504631003688872

Jones, M. L., \& Galliher, R. V. (2015). Daily racial microaggressions and ethnic identification among Native American young adults. Cultural Diversity and Ethnic Minority Psychology, 21, 1-9. doi:10.1037/a0037537

Juan, M. J. D., Syed, M., \& Azmitia, M. (in press). Intersectionality of race/ethnicity and gender among Women of Color and White Women. Identity.

Kang, S. K., \& Bodenhausen, G. V. (2015). Multiple identities in social perception and interaction: Challenges and opportunities. Annual Review of Psychology, 66, 547-574. doi:10.1146/annurev-psych-010814-015025

Kanji, S., \& Cahusac, E. (2015). Who am I? Mothers' shifting identities, loss and sensemaking after workplace exit. Human Relations, 68, 1415-1436. doi:10.1177/0018726714557336

Kidder, J. L. (2016). College republicans and conservative social identity. Sociological Perspectives, 59, 177-200. doi:10.1177/0731121415583104

Klein, O., Spears, R., \& Reicher, S. (2007). Social identity performance: Extending the strategic side of SIDE. Personality and Social Psychology Review, 11, 28-45. doi: $10.1177 / 1088868306294588$

Koch, K., \& Wann, D. L. (2014). Fans' identification and commitment to a sport team: The impact of self-selection versus socialization processes. In R. Schinke, R. Schinke (Eds.), Innovative writings in sport and exercise psychology (pp. 131-145). Hauppauge, NY, US: Nova Science Publishers. 
Luyckx, K., Schwartz, S. J., Rassart, J., \& Klimstra, T. A. (2016). Intergenerational associations linking identity styles and processes in adolescents and their parents. European Journal of Developmental Psychology, 13, 67-83. doi:10.1080/17405629.2015.1066668

Marcia, J. E. (1966). Development and validation of ego-identity status. Journal Of Personality And Social Psychology, 3(5), 551-558. doi:10.1037/h0023281

Martin, E. M., \& Horn, T. S. (2013). The role of athletic identity and passion in predicting burnout in adolescent female athletes. The Sport Psychologist, 27, 338-348.

McIntosh, P. (2012). Reflections and future directions for privilege studies. Journal of Social Issues, 68, 194-206. doi:10.1111/j.1540-4560.2011.01744.x

McAdams, D.P. (2001). The psychology of life stories. Review of General Psychology, 5, 100122.

McAdams, D. P., \& McLean, K. C. (2013). Narrative identity. Current Directions in Psychological Science, 22, 233-238.

McLean, K. C. (2015). The co-authored self: Family stories and the construction of personal identity. New York, NY, US: Oxford University Press.

McLean, K. C., \& Pratt, M. W. (2006). Life's little (and big) lessons: Identity statuses and meaning-making in the turning point narratives of emerging adults, Developmental Psychology, 42, 714-722.

McLean, K. C., \& Syed., M. (2015). Personal, master, and alternative narratives: An integrative framework for understanding identity development in context. Human Development, 58, 318-349.

McLean, K. C., Shucard, H., \& Syed, M. (2016). Gender Identity Development in Emerging Adulthood: A Master Narrative Approach. Emerging Adulthood. 
McLean, K. C., Syed, M., \& Shucard, H. (2016). Bringing Identity Content to the Fore: Links to Identity Development Processes. Emerging Adulthood.

McLean, K. C., Syed, M., Yoder, A., \& Greenhoot, A. (2016). The role of domain content in understanding identity development processes. Journal of Research on Adolescence, $26(1), 60-75$.

McNaughton, S. M., \& Billot, J. (2016). Negotiating academic teacher identity shifts during higher education contextual change. Teaching in Higher Education, 21, 644-658. doi:10.1080/13562517.2016.1163669

Meeus, W. (2011). The study of adolescent identity formation 2000-2010: A review of longitudinal research. Journal of Research on Adolescence, 21, 75-94.

Morgan, E. M., \& Davis-Delano, L. R. (2016). How public displays of heterosexual identity reflect and reinforce gender stereotypes, gender differences, and gender inequality. Sex Roles, doi:10.1007/s11199-016-0613-2

Nasir, N. S., \& Saxe, G. B. (2003). Ethnic and academic identities: A cultural practice perspective on emerging tensions and their management in the lives of minority students. Educational Researcher, 32(5), 14-18.

Pals, J. L. (2006). Authoring a second chance in life: Emotion and transformational processing within narrative identity. Research in Human Development, 3(2-3), 101-120. doi:10.1207/s15427617rhd0302\&3_3

Pasupathi, M., Mansour, E., \& Brubaker, J. R. (2007). Developing a life story: Constructing relations between self and experience in autobiographical narratives. Human Development, 50(2-3), 85-110. doi:10.1159/000100939

Purdie-Vaughn, V., \& Eibach, R. P. (2008). Intersectional invisibility: The distinctive advantages 
and disadvantages of multiple subordinate-group identities. Sex Roles, 59, 377-391.

Ragins, B. R., Singh, R., \& Cornwell, J. M. (2007). Making the invisible visible: Fear and disclosure of sexual orientation at work. Journal of Applied Psychology, 92, 1103-1118. doi:10.1037/0021-9010.92.4.1103

Richardson, B. L., Macon, T. A., Mustafaa, F. N., Bogan, E. D., Cole-Lewis, Y., \& Chavous, T. M. (2015). Associations of racial discrimination and parental discrimination coping messages with African American adolescent racial identity. Journal of Youth and Adolescence, 44, 1301-1317. doi:10.1007/s10964-014-0196-6

Rivas-Drake, D., Seaton, E. K., Markstrom, C., Quintana, S., Syed, M., Lee, R. M., \& ... Yip, T. (2014). Ethnic and racial identity in adolescence: Implications for psychosocial, academic, and health outcomes. Child Development, 85, 40-57. doi:10.1111/cdev.12200

Rivas-Drake, D., \& Witherspoon, D. (2013). Racial identity from adolescence to young adulthood: Does prior neighborhood experience matter? Child Development, 84, 19181932. doi:10.1111/cdev.12095

Rosenthal, L. (2016). Incorporating intersectionality into psychology: An opportunity to promote social justice and equity. American Psychologist,71(6), 474.

Rothenberg, P. S. (2016). White privilege: Essential readings on the other side of racism, $4^{\text {th }}$ edition. Worth Publishers: New York, NY.

Sanders Thompson, V. L. (1999). Variables affecting racial-identity salience among African Americans. The Journal of Social Psychology, 139, 748-761. doi:10.1080/00224549909598254

Schachter, E. P. (2004). Identity configurations: A new perspective on identity formation in contemporary society. Journal of Personality, 72(1), 167-200. 
Showers, C. J., \& Zeigler-Hill, V. (2007). Compartmentalization and integration: The evaluative organization of contextualized selves. Journal of Personality, 75(6), 1181-1204.

Stahl, G. (2016). White working-class male narratives of 'loyalty to self' in discourses of aspiration. British Journal of Sociology of Education, 37, 663-683. doi:10.1080/01425692.2014.982859

Schwartz, S. J., Zamboanga, B. L., Luyckx, K., Meca, A., \& Ritchie, R. A. (2013). Identity in emerging adulthood: Reviewing the field and looking forward. Emerging Adulthood, 1(2), 96-113.

Syed, M. (2010). Developing an integrated self: Academic and ethnic identities among ethnically-diverse college students. Developmental Psychology, 46(6), 1590-1604.

Syed, M. (2015). Theoretical and methodological contributions of narrative psychology to ethnic identity research. In C. Santos \& A. J. Umaña-Taylor (Eds.). Studying ethnic identity: Methodological advances and considerations for future research (pp. 27-54). Washington, DC: American Psychological Association.

Syed, M., \& Azmitia, M. (2010). Narrative and ethnic identity exploration: A longitudinal account of emerging adults' ethnicity-related experiences. Developmental psychology, 46(1), 208-219.

Syed, M., Azmitia, M., \& Cooper, C. R. (2011). Identity and academic success among underrepresented ethnic minorities: An interdisciplinary review and integration. Journal of Social Issues, 67(3), 442-468.

Syed, M., \& McLean, K. C. (2015). The Future of Identity Development Research: Reflections, Tensions, and Challenges. In K. C. McLean and M. Syed (Eds). The Oxford 
Handbook of Identity Development (pp. 562-574). New York: New York: Oxford University Press.

Szymanski, D. M., \& Carr, E. R. (2008). The roles of gender role conflict and internalized heterosexism in gay and bisexual men's psychological distress: Testing two mediation models. Psychology of Men \& Masculinity, 9(1), 40.

Tajfel, H. (1978). Differentiation between social groups: Studies in the social psychology of intergroup relations. Oxford, England: Academic Press, 1978.

Thomas, V., \& Azmitia, M. (2016). Tapping Into the App Updating the Experience Sampling Method for the 21st Century. Emerging Adulthood, 4(1), 60-67.

Toomey, R. B., Anhalt, K., \& Shramko, M. (2016). An examination of the validity and reliability of a measure of sexual orientation identity exploration, resolution, and affirmation. Self and Identity, 15, 488-504. doi:10.1080/15298868.2016.1157516

Tsang, S. M., Hui, E. P., \& Law, B. M. (2013). Positive identity as a positive youth development construct: A conceptual review. In D. L. Shek, R. F. Sun, J. Merrick, D. L. Shek, R. F. Sun, J. Merrick (Eds.), Positive youth development: Theory, research and application (pp. 79-92). Hauppauge, NY, US: Nova Science Publishers.

van Vuuren, M., Teurlings, J., \& Bohlmeijer, E. (2012). Shared fate and social comparison: Identity work in the context of a stigmatized occupation. Journal Of Management \& Organization, 18(2), 263-280. doi:10.5172/jmo.2012.18.2.263

Verkuyten, M. (2016). Further conceptualizing ethnic and racial identity research: The social identity approach and its dynamic model. Child Development, 87, 1796-1812.

Waterman, A. S. (2015). What does it mean to engage in identity exploration and to hold identity commitments? A methodological critique of multidimensional measures for the study of 
identity processes. Identity: An International Journal of Theory and Research, 15, 309349. doi:10.1080/15283488.2015.1089403

Weststrate, N.,\& McLean, K. C. (2010). The rise and fall of gay: Gay identity development in different historical and cultural cohorts. Memory, 18, $225-240$.

Yap, S. Y., Donnellan, M. B., Schwartz, S. J., Zamboanga, B. L., Kim, S. Y., Huynh, Q., \& ... Brown, E. J. (2016). Evaluating the invariance of the Multigroup Ethnic Identity Measure across foreign-born, second-generation and later-generation college students in the United States. Cultural Diversity and Ethnic Minority Psychology, 22, 460-465. doi:10.1037/cdp0000068

Yip, T. (2005). Sources of situational variation in ethnic identity and psychological well-being: A palm pilot study of Chinese American students. Personality and Social Psychology Bulletin, 31(12), 1603-1616.

Yip, T., Seaton, E. K., \& Sellers, R. M. (2006). African American racial identity across the lifespan: Identity status, identity content, and depressive symptoms. Child Development, 77, 1504-1517. doi:10.1111/j.1467-8624.2006.00950.x 


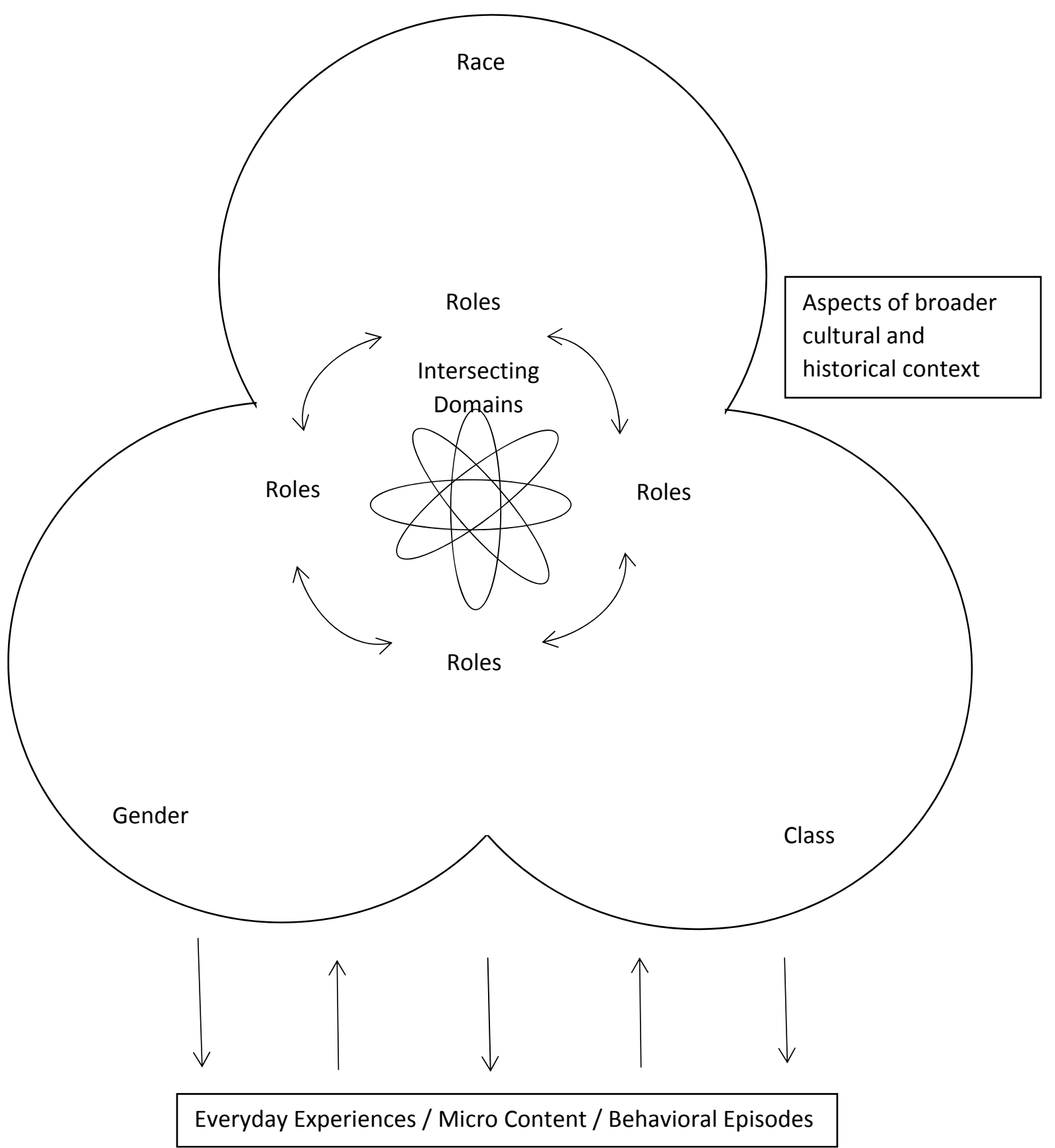

Figure 1. A multilevel model of identity content in context. 